\title{
Why Acceleration?
}

\author{
Bernard J. Feldman \\ University of Missouri-St. Louis, St. Louis, USA
}

\begin{abstract}
This paper is a suggested lecture to introduce forces and accelerations in an introductory engineering physics class. It explains why acceleration is at the heart of physics using the ideas of Galilean transformations and the invariance of acceleration, where forces come from using experimental observations of the accelerations of the moon, an apple, charged balloons, iron magnets, protons, and neutrons, and then goes onto a brief outline of the modern view of forces between fundamental particles by introducing the ideas of virtual particles and field theory.
\end{abstract}

Keywords: acceleration, force, inertial frames of reference, invariance

\section{Introduction}

At the heart of most first semester introductory physics courses are Newton's three laws and the pivotal role played by acceleration. The study of mechanics is just the study of accelerations. Most textbooks really do not adequately address the question: Why is acceleration at the heart of mechanics? In this paper, this question will be answered in a way that most high school and college students can comprehend.

\section{Acceleration}

The goal of mechanics is to describe and then explain motion. However, there are two fundamental requirements of any such explanation. The first is that any explanation must be universal, namely, a physics textbook must be valid everywhere in the universe and at any velocity.

The second requirement is that the relative motion problem be solved. The relative motion problem is- How do you know if an object is moving or accelerating or not. For example, if a rock is dropped from the top of a building, the rock is accelerating with respect to a person in the building, but in the frame of reference of a person sitting on the rock, the rock is at rest.

The solution to both the relative motion problem and the universal requirement is to only do physics in inertial frames of reference - frames of reference at rest or at a constant velocity relative to distant stars. Question for your students: Is your classroom an inertial frame of reference? You will be surprised by how many students say "Yes". However, given that the acceleration of your classroom is on the order of $0.01 \mathrm{~m} / \mathrm{sec}^{2}$ due to the earth's rotation about its axis and about the sun, to a good approximation, your classroom is an inertial frame of reference (Tipler \& Mosca, 2008).

Measurements in one inertial frame of reference can then be transformed into measurements in any other inertial frame of reference moving at a velocity $u$ with respect to the first frame of reference by using the Galilean transformation:

$$
r^{\prime}=r-u t
$$

Bernard J. Feldman, professor, Department of Physics and Astronomy, University of Missouri-St. Louis. 


$$
t^{\prime}=t
$$

where $r$ is the position vector of an object at time $t$ in the first inertial frame of reference, and $r$ ' is the position vector of the object at time $t^{\prime}$ in the second inertial frame of reference. It should be noted that it is assumed that the clocks in both inertial frames of reference are synchronized with each other (Also, at $t=0$, the two coordinate systems overlap, namely, $r^{\prime}=r$ ).

The velocity vector of the object in the second frame of reference is:

$$
v^{\prime}=d r^{\prime} / d t^{\prime}=\left(d r^{\prime} / d t\right)\left(d t / d t^{\prime}\right)=d(r-u t) / d t=d r / d t-u=v-u
$$

where $v$ is the velocity vector of the object in the first frame of reference. The important result comes from taking a second derivative of $r$ ' with respect to $t$ ' to derive that the acceleration vector of the object in the second frame of reference is:

$$
a^{\prime}=d v^{\prime} / d t^{\prime}=\left(d v^{\prime} / d t\right)\left(d t / d t^{\prime}\right)=d(v-u) / d t=d v / d t=a
$$

where $d u / d t=0$, since $u$ is a constant. The acceleration vector of the object in the second frame of reference, $a^{\prime}$, is equal to the acceleration vector of the object in the first frame of reference, $a$. Not only is an object that accelerates in one inertial frame of reference accelerating in every other inertial frame of reference-the solution to the relative motion problem - but the acceleration is the same in all inertial frames of reference (invariant) — the requirement for a universal physics.

It is incumbent to tell you that what you already know and what most students do not know, namely, that Einstein's theory of special relativity proves that the above discussion is all incorrect. One must use Lorentz transformations, not Galilean transformations, to move from one inertial frame of reference to another, and under Lorentz transformations, the acceleration is not invariant. However, for velocities small compared to the speed of light, the invariance of the acceleration vector is a very good approximation. A brief summary of a separate lecture introducing special relativity and the Lorentz transformations can be found in the Appendix.

Now, with that background, you are ready to apply this idea of a physics of acceleration to a variety of situations. The first situation to be discussed is an imaginary scenario involving Isaac Newton sitting under an apple tree and looking east at a rising moon. The students were asked what thought went through Isaac Newton's head the instant an apple fell on his head. After a number of embarrassing answers, the class realized that both the apple and the moon are accelerating toward the center of the earth. Are the magnitudes of the accelerations of the apple and the moon the same? The class was reminded that they have covered rotational motion and that they can calculate the moon's radial acceleration, $a_{r}$, namely:

$$
a_{r}=\omega^{2} r
$$

where $\omega$ is the moon's angular velocity and $r$ is the distance from the earth to the moon. As part of this calculation, ask your class how long does it take the moon to make one rotation about the earth? You will be quite amused at all the embarrassing wrong answers. Using $r=3.84 \times 10^{-6} \mathrm{~m}$ and $\omega=6.28 / 27.3$ days, $a_{r}=2.7 \times$ $10^{-3} \mathrm{~m} / \mathrm{sec}^{2}$. It should be noticed that the sidereal month of the moon of 27.3 days was used, rather than the synodic month of 29.5 days. The sidereal month of the moon is measured with respect to distant stars-an inertial frame of reference. The synodic month of the moon is measured with respect to the earth-a non-inertial frame of reference (The StarChild Team, n.d.).

This led Newton to the idea that the earth is somehow grabbing both the apple and the moon-what we call the gravitational force. This was a revolutionary idea in Newton's time, namely, that the same laws of physics apply to the heavens - God's perfect creation — and the earth — the world of sin and corruption. 


\section{Forces}

The history of physics can be described as the following: An acceleration is observed first and then a force is inferred from the observation of that acceleration. For example, balloons that are charged by rubbing with a cloth or iron objects that are magnetized by a magnetic field are observed to attract or repel each other. The measured accelerations of those objects are modeled by the electromagnetic force. The nucleus of an atom is observed by Rutherford scattering to contain numerous protons. The fact that those protons do not fly apart from electromagnetic repulsion suggests that there must be a stronger, attractive nuclear force- the nuclear force. The neutron is observed to beta decay into a proton, an electron, and an antineutrino. Since a free neutron is electrically neutral and not influenced by either the nuclear or electromagnetic force, it is concluded that it is the weak force that governs that decay.

\section{Modern Viewpoint}

Finally, a difficult question was asked in the class: How does one particle exert a force on another particle - the force at a distance question. How does the earth down there grab the apple and the moon up here? You can then very briefly summarize the two complementary views from modern physics - virtual particles and field theory. There is a virtual particle associated with each fundamental force - a gluon for the nuclear force, a photon for the electromagnetic force, an intermediate vector boson for the weak force, and a graviton for the gravitational force. All these virtual particles have no mass and integer spin (Any particle with an integer spin is called a Bose particle) and exert force by carrying momentum between the fundamental particles, quarks, and leptons, that all have mass and half integer spin (Any particle with a half integer spin is called a Fermi particle). This clearly suggests the unification of these four forces into one force at the earliest times of the big bang - the grand unification theory (for an excellent introductory treatment of particle physics) (Walker, 2008).

Sometimes, the students ask what is spin. They were told that they can think of these fundamental particles as little magnets or as little current loops. Furthermore, just as every electron has the same mass and the same electrical charge, every electron has the same spin. Mass, electrical charge, and spin are all quantized. Some of the students may be acquainted with Pauli exclusion principle from chemistry, namely, that each electron must be in a different quantum state. All Fermi particles obey the Pauli exclusion principle, but all Bose particles do not. This discussion is a good way to expose your students to the beginnings of quantum statistics.

The second view is the field view, namely, that particles are not singularities but fields that extent throughout all space. Thinking of each proton as a mini funnel, two protons repel each other when their funnels overlap each other. In the case of the earth, the apple, and the moon, the gravitational funnel of the earth overlaps the apple and the moon, giving rise to their accelerations towards the center of the earth, and the gravitational funnel of the moon overlaps the earth, giving rise to the ocean tides on earth.

\section{Conclusion}

This paper is a summary of a lecture to introduce forces and accelerations in a beginning engineering physics class. It explains why acceleration is at the heart of physics, where forces come from, and then goes onto a brief outline of the modern view of forces between fundamental particles. It introduces students to Galilean transformations, invariance, the four forces in nature (strong, electromagnetic, weak, and gravitational), and the modern concepts of virtual particles, Bose and Fermi statistics, and field theory. This 
lecture provides an overview and framework for all the physics that will come in all their later physics courses.

\section{References}

Serway, R. A., \& Jewett, Jr., J. W. (2004). Physics for scientists and engineers (6th ed., p. 1244). Belmont, C.A.: Thomson, Brooks/Cole.

The StarChild Team. (n.d.). StarChild question of the month for April 2001. Retrieved from http://starchild.gsfc.nasa.gov/docs/ StarChild/questions/question32.html

Tipler, P. A., \& Mosca, G. (2008). Physics for scientists and engineers (6th ed., Volume 1, p. 94). New York, N.Y.: W. H. Freeman and Company.

Walker, J. (2008). Halliday/Resnick fundamentals of physics (Extended, 8th ed., p. 1219). Hoboken, N.J.: John Wiley \& Sons. 


\section{Appendix: Summary of a Separate Lecture Introducing Special \\ Relativity and the Lorentz Transformations}

At a later date, the author recommends a lecture introducing the ideas of special relativity (for an excellent introductory treatment of special relativity) (Serway \& Jewett, 2004). The author starts with the experimental result of Michelson and Morley, namely, that the speed of light, $c$, is invariant, namely, it equals $3 \times 10^{8} \mathrm{~m} / \mathrm{sec}$ in all inertial frames of reference. He then defines a light clock and derives the time dilation equation of a moving clock. He asks his class how does a person in a moving frame of reference sees your stationary clock? That moving person sees your stationary clock going slow. How can that be? Now, the author introduces the idea of non-synchronous clocks, namely, two clocks in your frame of reference are synchronous (reading the same time) but a person in another moving frame of reference sees your two synchronous clocks as non-synchronous (reading different times). If two moving clocks coming towards you, the one nearest you is at a later time than the one farther away from you. Combining time dilation with non-synchronous clocks resolves the time dilation paradox.

One can then point out that two simultaneous events in one frame of reference are two non-simultaneous events in another frame of reference. In other words, two events at different places, but at the same time in one frame of reference, transform into two events at different places and at different times in a moving frame of reference. Generalizing, space-time transforms into space-time. This leads to the idea of four-vectors and the invariance of the magnitude of all four-vectors. Finally, the author introduces the energy-momentum four-vector and Einstein's famous equation. He points out that conservation of energy and the conservation of linear momentum are now one law - the conservation of the four components of the energy-momentum four-vector. And one can include all forms of potential energy into the mass of the relativistic energy formula.

Although it is not recommended to teach the Lorentz transformations in an introductory class, they are included here for the sake of completeness. The Lorentz transformations are:

$$
\begin{gathered}
x^{\prime}=\chi(x-u t) y^{\prime}=y z^{\prime}=z \\
t^{\prime}=\chi\left(t-u x / c^{2}\right)
\end{gathered}
$$

where $x, y$, and $z$ are the components of the position vector in the stationary frame of reference, $x^{\prime}, y^{\prime}$, and $z^{\prime}$ are the components of the position vector $r$ ' in the moving frame of reference. The moving frame of reference is traveling with a velocity $u$ in the $x$ direction with respect to the stationary frame of reference, and $\gamma=\left(1-u^{2} / c^{2}\right)^{-1 / 2}$.

It should be noted that the Lorentz transformations explicitly show that two events at $x=0, t=0$, and $x=x, \mathrm{t}=0$ that are at the same time in the stationary frame of reference are at different times in the moving frame of reference, $x^{\prime}=0, t^{\prime}=0$, and $x^{\prime}=\gamma^{\prime}$, $t^{\prime}=-\mu u x / c^{2}$. Also, time dilation of a moving clock is easily seen by transforming a moving clock which is first at $x=0, t=0$, and later at $x=u t, t=t$. The transformations of these two events are $x^{\prime}=0, t^{\prime}=0$ and $x^{\prime}=0, t^{\prime}=\gamma^{-1} t$, where $\gamma^{-1} t$ is less than $t$ - the one moving clock is slow compared to the two synchronized stationary ones.

The invariant quantities in special relativity are the magnitudes of the four vectors. In the case of the space-time four-vector:

$$
x^{2}+y^{2}+z^{2}-c^{2} t^{2}=x^{2}+y^{2}+z^{2}-c^{2} t^{2}
$$

It should be noticed from this equation that the invariance of the speed of light is obvious. Any object moving from $x=y=z$ $=t=0$ to $x^{2}+y^{2}+z^{2}=c^{2} t^{2}$ is traveling at the speed of light in all inertial frames of reference. 